\title{
Tratamiento de infecciones fúngicas sistémicas. III parte: Anfotericina B, aspectos farmacoeconómicos y decisiones terapéuticas
}

\author{
ALBERTO FICA C.
}

\author{
Treatment of systemic fungal infections. III Part: Amphotericin B, \\ pharmacoeconomical aspects and therapeutic decisions
}

This third part contains a review on amphotericin B, pharmacoeconomical aspects and clinical decision involved during therapy selection of antifungal agents. Amphotericin B, the standard of antifungal therapy is associated with significant infusion adverse events and nephrotoxicity but has the widest spectrum of activity against fungal species involved in human infections. It is a recognized first line-therapy in high-risk patients with criptocococcal meningitis, invasive aspergillosis, mucormicosis, and as an empirical therapy in persistently febrile neutropenic patients without renal failure. Several lipid associated formulations of amphotericin B are available that provide lesser nephrotoxicity but at a considerable higher cost and an adverse cost-effectiveness ratio. Several small-randomized controlled trials of Intralipid ${ }^{\circledR}$ formulations of amphotericin B have been published but controversy remains. Few pharmacoeconomical analysis have been published and mostly refer to lipid associated formulations of amphotericin B versus the conventional form. As stated, potential economical benefits obtained with lipid associated formulations of amphotericin B by avoiding more cases of renal failure are surpassed by their enormous acquisition costs. On the other hand, amphotericin B is a cost-effective alternative compared to a prolonged iv fluconazol therapy but not when switching to oral therapy is scheduled before the first week. Intravenous itraconazole (not available in Chile) is reportedly more cost-effective than conventional amphotericin B for the treatment of persistently febrile neutropenic patients.

Key words: Amphotericin B deoxycolate; Lipid associated formulations of amphotericin B; Fungal infections, Treatment.

Palabras claves: anfotericina B deoxicolato; Formulaciones de anfotericina B asociadas a lípidos; Infecciones fúngicas; Tratamiento.

\section{Introducción}

Diferentes compuestos antifúngicos se encuentran disponibles y validados para el tratamiento de infecciones fúngicas sistémicas o invasoras (Tabla 1). Las alternativas terapéuticas habituales representadas por fluconazol y anfotericina B deoxicolato ("convencional"), se han expandido con otros compuestos triazoles o formulaciones parenterales de ellos, formulaciones de anfotericina B asociadas a lípidos y equino- candinas. El propósito de esta revisión es orientar al lector sobre las ventajas y desventajas de la anfotericina B convencional y sus formulaciones asociadas a lípidos, junto a los aspectos farmacoeconómicos involucrados en el uso de las terapias antifúngicas sistémicas. Este artículo complementa una publicación previa sobre compuestos triazólicos ${ }^{1}$ y otro sobre caspofungina y otras equinocandinas ${ }^{2}$. La flucitosina no se incluye en esta revisión por su papel secundario.

Sección Infectología

Hospital Clínico Universidad de Chile

Recibido: 29 septiembre 2003

Aceptado: 2 abril 2004 
Tabla 1. Compuestos antifúngicos sistémicos disponibles y mecanismo de acción

\begin{tabular}{ll}
\hline Compuesto & Mecanismo de acción \\
\hline $\begin{array}{l}\text { Triazólicos } \\
\text { - Fluconazol } \\
\text { - Itraconazol } \\
\text { - Voriconazol }\end{array}$ & $\begin{array}{l}\text { Inhiben la enzima lanosterol 14-alfa demetilasa, bloqueando la síntesis } \\
\text { final de ergosterol, compuesto esencial de la membrana celular de los } \\
\text { hongos. Efecto fundamentalmente fungistático, aunque voriconazol } \\
\text { exhibe una acción fungicida contra el género Aspergillus }\end{array}$ \\
$\begin{array}{l}\text { Polienos } \\
\text { - Anfotericina B } \\
\text { - Formulaciones de anfotericina B } \\
\text { asociadas a lípidos }\end{array}$ & $\begin{array}{l}\text { Interactúa directamente con el ergosterol de la membrana celular fúngica } \\
\text { No interfiere con la síntesis de la membrana sino que la desestabiliza }\end{array}$ \\
$\begin{array}{l}\text { Equinocandinas } \\
\text { E Caspofungina }\end{array}$ & $\begin{array}{l}\text { Inhibe enzima beta (1,3)-glucano sintetasa involucrada en la síntesis } \\
\text { de la pared celular de los hongos. Efecto fungicida por lisis sobre }\end{array}$ \\
& Candida spp \\
\hline
\end{tabular}

Tabla 2. Susceptibilidad in vitro de diferentes especies de hongos filamentosos a compuestos triazólicos, anfotericina B y caspofungina. Valores expresados en $\mathrm{CIM}_{90}$ o media geométrica $(\mu \mathrm{g} / \mathrm{ml})$

\begin{tabular}{llccc}
\hline Especie & $\begin{array}{c}\text { Itraconazol } \\
\mathbf{C I M}_{\mathbf{9 0}}\end{array}$ & $\begin{array}{c}\text { Voriconazol } \\
\mathbf{C I M}_{\mathbf{9 0}}\end{array}$ & $\begin{array}{c}\text { Anfotericina B } \\
\mathbf{C I M}_{\mathbf{9 0}}\end{array}$ & $\begin{array}{c}\text { Caspofungina } \\
\text { Media } \\
\text { geométrica } \\
\text { o CIM }\end{array}$ \\
\hline A. fumigatus & $0,5-1$ & $0,25-2$ & $0,5-4$ & $0,19-2,15$ \\
A. flavus & $0,25-1$ & $0,5-2$ & $0,5-8$ & $0,5-2,75$ \\
A. niger & $0,5-4$ & $0,5-4$ & $0,125-4$ & $0,04-0,4$ \\
A. terreus & $0,125-0,25$ & 1 & $4->16$ & $0,2-0,5$ \\
Scedosporium apiospermum & $4->16$ & $0,25-2$ & $8->16$ & $4-8$ \\
Fusarium solani & $>16$ & $4->8$ & 2 & -4 \\
\hline
\end{tabular}

Datos tomados de referencias $1,3,4,5$.

\section{Anfotericina B convencional}

La anfotericina B deoxicolato es un compuesto poliénico cuyo efecto antifúngico se logra mediante la interacción directa con el ergosterol de la membrana celular fúngica (Tabla 1). Anfotericina B no interfiere con la síntesis de la membrana sino que la desestabiliza, facilitando la formación de canales con la pérdida consecuente de iones y componentes celulares. Inhibe una bomba de protones en Candida albicans e induce la peroxidación de los lípidos de membranas ${ }^{3}$. Este compuesto tiene el mayor espectro de acción antifúngico conocido, incluyendo levaduras como Candida y Cryptococcus y hongos filamentosos como Aspergillus, Rhizopus spp y Fusarium spp (Tabla 2). No tiene actividad contra Candida lusitaniae, Trichosporon spp, Geotrichum spp, Scedosporium apiospermum, Pseudallescheria boydii, Aspergillus terreus y los agentes de dermatomicosis ${ }^{3}$. Anfotericina B ejerce al igual que caspofungina un efecto postantifúngico mayor a 12 horas de duración contra Candida spp $^{6}$.
Anfotericina B es una herramienta terapéutica de demostrada utilidad en el manejo de diferentes infecciones fúngicas o en el tratamiento empírico de pacientes con una infección probable, como: candidiasis sistémica, aspergilosis invasora pulmonar o extrapulmonar, criptococosis meníngea, mucormicosis y en el manejo de pacientes neutropénicos febriles sin respuesta al tratamiento antibacteriano. En este último grupo, los resultados de un meta-análisis sugieren que anfotericina B podría ser el único preparado asociado a una disminución de la mortalidad de esta condición, a diferencia de otros compuestos antifúngicos ${ }^{7}$. Anfotericina B tiene una utilidad muy limitada para el tratamiento de las infecciones por $A$. terreus, Trichosporon beigelii y P. boydii.

Limitaciones. A pesar de su espectro de acción privilegiado, anfotericina $\mathrm{B}$ tiene varias limitaciones terapéuticas:

- Eficacia clínica subóptima para el manejo de infecciones fúngicas invasoras, especialmente en aspergilosis (no supera el 40 a 50\%). Por 
otra parte, los resultados obtenidos en infecciones extrapulmonares, especialmente en el SNC, donde su penetración es limitada, son muy reducidos. Esta eficacia no parece aumentar con un incremento de las dosis o con el uso de las formulaciones asociadas a lípidos ${ }^{8}$.

- La severidad y frecuencia de las reacciones adversas. La infusión endovenosa se asocia a fiebre y calofríos y otros efectos tardíos como nefrotoxicidad, hipokalemia y acidosis metabólica. La frecuencia de estas manifestaciones supera el 20 a $30 \%$, obliga a incorporar premedicaciones frecuentes que incluyen corticosteroides y, en ocasiones, impiden continuar con el tratamiento ${ }^{8}$. En el desarrollo de la toxicidad renal participan también otros factores como el uso concomitante de otros fármacos nefrotóxicos y la depleción de volumen, pero no parece depender de la dosis acumulada de anfotericina $\mathrm{B}^{8}$. La filtración glomerular decae en aproximadamente $40 \%$ en las primeras dos semanas y luego se estabiliza a 20 a $60 \%$ del valor normal hasta el fin del tratamiento, no constituyendo un cambio irreversible en la mayor parte de los pacientes ${ }^{8,9}$.

Control de los efectos adversos. Dos estrategias se han delineado para controlar los efectos adversos asociados al uso de anfotericina B deoxicolato. Estas estrategias involucran por una parte, modificaciones en la forma de administración de la misma, lo que incluye precargas salinas, aporte de potasio o administración del medicamento en 24 horas en lugar de las 4 horas convencionales ${ }^{10,11}$. La otra alternativa es el reemplazo por formulaciones asociadas a lípidos.

La estrategia de aplicar en forma lenta la anfotericina B convencional logró demostrar una reducción significativa de los efectos adversos al comparar una infusión en 24 horas versus 4 horas ${ }^{11}$. La frecuencia de fiebre durante el primer día se redujo de 53 a $25 \%$, la de calofríos de 53 a $13 \%$ y la de vómitos de 35 a 10\%. También se observó una reducción de las tasas de calofríos y vómitos en todo el período de estudio, al igual que en el consumo de corticosteroides, opiáceos o paracetamol como premedicación. La tasa de interrupciones de tratamiento disminuyó significativamente desde 28 a $8 \%$ y los valores de PCR aumentaron en una menor proporción de pacientes. La frecuencia de casos con aumento de creatininemia fue ostensiblemente menor al igual que el desarrollo de acidosis metabólica ${ }^{11}$. Se ha reportado además que no existen diferencias significativas entre una infusión en 1 $y$ otra en 4 horas $^{12}$.
La administración previa de una carga salina diaria de $1.000 \mathrm{ml}$ antes de recibir la dosis de anfotericina B convencional, también ha demostrado ser en forma independiente, un factor protector de daño renal aunque asociado a una mayor pérdida de potasio. La frecuencia de pacientes que presentaron una duplicación de la creatininemia fue de $40 \%$ para el grupo no prehidratado versus $0 \%$ para el grupo que recibió precarga salina, aunque estos datos fueron obtenidos en pacientes tratados por leishmaniasis, que no representan los pacientes médico-quirúrgicos habituales ${ }^{13}$. Antes o durante el tratamiento se debe evaluar si el paciente está depletado de volumen, si puede tolerar una precarga salina o si evoluciona con pérdidas de volumen importante por fiebre o vómitos que pudieran acentuar la nefrotoxicidad de anfotericina $\mathrm{B}^{10}$.

Formulaciones de anfotericina $B$ asociadas a lípidos: han intentado mejorar la solubilidad de las moléculas apolares de anfotericina B convencional, originalmente dispersas en deoxicolato, por moléculas lipídicas con cabezas polares. El objetivo primario de estos cambios es disminuir la toxicidad renal, y no, mejorar la eficacia clínica del producto. Las formulaciones asociadas a lípidos difieren en el tamaño y estructura de sus moléculas, las concentraciones máximas alcanzadas, el área bajo la curva de concentración en el plasma ( $\mathrm{ABC}$ ), las reacciones adversas asociadas y su capacidad de penetrar a ciertos tejidos específicos como el $\mathrm{SNC}^{3}$. En la Tabla 3 se indican algunas de estas propiedades. Estos preparados se han evaluado entre sí y también entre la formulación convencional. Diferentes estudios demuestran que las formulaciones asociadas a lípidos presentan una eficacia clínica similar a anfotericina B deoxicolato para el tratamiento de pacientes neutropénicos con sospecha de infección fúngica o de pacientes con aspergilosis invasora o criptococosis meníngea ${ }^{9,14}$, y demuestran en forma convincente una reducción de $50 \%$ o más en la tasa de nefrotoxicidad. Sin embargo, en estos mismos estudios se ha demostrado que algunas formulaciones asociadas a lípidos presentan reacciones adversas significativas que equiparan la frecuencia de interrupciones de tratamiento observadas con la forma convencional o incluso presentan una mayor frecuencia de fiebre y calofríos (Amphotec ${ }^{\circledR} /$ Amphocil $\left.{ }^{\circledR}\right)^{14}$. En una comparación directa entre AmBisome ${ }^{\circledR}$ (liposomal) y Abelcet ${ }^{\circledR}$ (complejo lipídico), se demostró que este último se asoció significativamente a una mayor frecuencia de fiebre, calofríos, hipoxia y nefrotoxicidad en comparación a la presentación liposomal ${ }^{9}$. 
Tabla 3. Características de formulaciones de anfotericina $B$ asociadas a lípidos

\begin{tabular}{|c|c|c|c|c|c|c|}
\hline Preparado & Formulación & $\begin{array}{c}\text { Dosis } \\
\text { habitual } \\
\text { (mg/kg) }\end{array}$ & $\begin{array}{l}\text { Cmax } \\
\mu \mathrm{g} / \mathrm{ml}\end{array}$ & Nefrotoxicidad & $\begin{array}{c}\text { Reacciones } \\
\text { adversas }\end{array}$ & $\begin{array}{c}\text { Penetración } \\
\text { al SNC* } \\
(\mu \mathrm{g} / \mathrm{g})\end{array}$ \\
\hline Anfotericina B deoxicolato & & 0,6 & 1,1 & +++ & +++ & 0,18 \\
\hline AmBisome $\AA$ & Liposomal & 5 & 83 & + & + & 1,99 \\
\hline Abelcet ${ }^{\circledR}$ & $\begin{array}{l}\text { Complejo } \\
\text { lipídico }\end{array}$ & 5 & 1,7 & + & $+++* *$ & 0,27 \\
\hline Amphocil®/Amphotec $®$ & $\begin{array}{c}\text { Dispersión } \\
\text { coloidal }\end{array}$ & 5 & 3,1 & + & $+++* * *$ & 0,22 \\
\hline
\end{tabular}

*: concentraciones cerebrales en un modelo animal. Dosis de $1 \mathrm{mg} / \mathrm{kg}$ (ref 9); **: mayor frecuencia de fiebre, calofríos y nefrotoxicidad que AmBisome $® ; * * *$ : tasa similar de suspensión de tratamiento que anfotericina B convencional y una tasa superior de fiebre y calofríos.

Tabla 4. Resumen propiedades e indicaciones de las formulaciones de anfotericina $B$ asociadas a lípidos

Eficacia similar a la anfotericina deoxicolato (convencional)

Menor nefrotoxicidad

Reacciones adversas sistémicas asociadas a productos específicos

Alto costo

Indicadas en pacientes con infecciones fúngicas sistémicas, que presentan intolerancia o refractariedad a tratamiento con anfotericina $\mathrm{B}$ deoxicolato o ante falla renal preexistente
Las formulaciones asociadas a lípidos son una alternativa terapéutica de alto costo que puede ser considerada cuando se presentan reacciones adversas asociadas a la infusión, falla renal progresiva o refractariedad a una dosis acumulada de $500 \mathrm{mg}$ de anfotericina B convencional. También son consideradas una alternativa para el tratamiento de infecciones fúngicas demostradas o probables en pacientes con falla renal establecida ${ }^{15}$ (Tabla 4).

En la Tabla 5 se presenta un resumen de los aspectos más importantes en el manejo de anfotericina B convencional.

Anfotericina B como emulsión lipídica en Intralipidß. Esta mezcla se ha diseñado con el objetivo de reducir el costo de estos preparados.

Varias exigencias deben cumplirse en su preparación e incluyen el uso de concentraciones de

Tabla 5. Manejo de anfotericina $B$ en el escenario clínico

- Muchos expertos opinan que la aplicación en dosis progresivas no es necesaria

- Corregir cualquier estado de depleción de volumen en el paciente antes o durante el tratamiento

- Minimizar la aplicación de otros fármacos nefrotóxicos

- Aplicar una precarga salina de $1.000 \mathrm{~mL}$ (o menor de acuerdo a las condiciones del paciente)

- Aplicar anfotericina B en una dosis continua en 24 horas

- No aplicar rutinariamente antipiréticos o corticosteroides como medida profiláctica para disminuir las reacciones adversas asociadas a la infusión. Utilizarlas de acuerdo a la aparición de reacciones adversas

- Monitorizar periódicamente la función renal y los valores de potasio y magnesio en la sangre.

- En caso de desarrollo de falla renal, y si las condiciones del paciente lo permiten, considerar una interrupción temporal del tratamiento o un reemplazo por otro compuesto (caspofungina, anfotericina B liposomal, voriconazol o itraconazol solución oral) 
Tabla 6. Costo comparativo de diferentes preparados antifúngicos en E.U.A.

\begin{tabular}{lcc}
\hline Compuesto & Dosis diaria & $\begin{array}{c}\text { Costo diario en US\$ } \\
\text { para tratar a un paciente de 70 kg }\end{array}$ \\
\hline Polienos & $(\mathrm{mg} / \mathrm{kg})$ & $18-36$ \\
Anfotericina B deoxicolato & 0,5 a 1 & $565-942$ \\
Anfotericina B complejo lipídico (Abelcet®) & 5 & $446-480$ \\
Anfotericina B dispersión coloidal (Amphotec ()$)$ & $3 \mathrm{a} 4$ & $942-1.319$ \\
Anfotericina liposomal (AmBisome®) & $3 \mathrm{a} 6$ & \\
Equinocandinas & $(\mathrm{mg})$ & $288-371$ \\
Caspofungina & $50-70$ & $133-266$ \\
Fluconazol & $(\mathrm{mg})$ & $26-52$ \\
Endovenoso & $400 \mathrm{a} 800$ & \\
Oral & $400 \mathrm{a} 800$ & $14-28$ \\
Itraconazol & $(\mathrm{mg})$ & $16-33$ \\
Cápsulas orales & $200 \mathrm{a} 400$ & $176-352$ \\
Solución oral & $200 \mathrm{a} 400$ & \\
Endovenoso & $200 \mathrm{a} 400$ & \\
\hline
\end{tabular}

lípidos no superiores a $20 \%$, concentraciones finales de anfotericina $\mathrm{B} \leq 1 \mathrm{mg} / \mathrm{ml}$ y un tiempo de administración de al menos una hora. Las emulsiones preparadas por agitación manual deben ser administradas inmediatamente. Aquellas preparadas con una agitación vigorosa y prolongada (18 horas), permiten generar una emulsión estable que evita la separación en varias fases luego de la preparación ${ }^{16,17}$. La actividad antifúngica de los preparados mezclados manualmente decae a las dos semanas de almacenamiento a $4^{\circ} \mathrm{C}$, en comparación a la estabilidad de los preparados mezclados por una agitación enérgica. La emulsión con Intralipid ${ }^{\circledR}$ utilizada a dosis equivalentes $(1 \mathrm{mg} / \mathrm{kg}$ ) se asocia a una menor Cmax y $\mathrm{ABC}$ en comparación a los valores obtenidos con anfotericina $\mathrm{B}$ deoxicolato ${ }^{3,18}$.

Al menos diez estudios randomizados han com- parado las reacciones adversas asociadas a la infusión y la nefrotoxicidad de anfotericina B en solución glucosada o en Intralipid®. Los resultados clínicos obtenidos han sido discordantes ${ }^{19-28}$. Parte de estas discrepancias se originan en las diferentes concentraciones de los preparados, en el uso concomitante de fármacos nefrotóxicos, en las definiciones de toxicidad, en el momento utilizado para el análisis o en las dosis totales aplicadas $^{29}$. A pesar de que no está disponible un meta-análisis formal, la combinación de anfotericina B en Intralipid® se ha asociado en 7 de 10 estudios a una menor tasa de efectos adversos durante la infusión (fiebre o calofríos) y en 6 de ellos a menos nefrotoxicidad, aunque en un estudio se señala una mayor frecuencia de eventos respiratorios graves para esta formulación. Sin embargo, hasta ahora el número total de pacientes

Tabla 7. Costo de antifúngicos sistémicos en Chile

\begin{tabular}{lcc}
\hline Nombre compuesto y presentación & $\begin{array}{c}\text { Dosis habitual de } \\
\text { mantención diaria } \\
\text { para un paciente de 70 kg }\end{array}$ & Costo diario de tratamiento* \\
\hline Fluconazol $200 \mathrm{mg}$ comprimidos & $400 \mathrm{mg}$ & 15.416 \\
Fluconazol $200 \mathrm{mg}$ ampollas & $400 \mathrm{mg}$ & 47.312 \\
Voriconazol endovenoso** & $600 \mathrm{mg}$ & 280.000 \\
Voriconazol oral** & $600 \mathrm{mg}$ & 60.000 \\
Anfotericina B deoxicolato 50 mg & $0,7 \mathrm{mg} / \mathrm{kg}^{* *}$ & 57.223 \\
Anfotericina B liposomal 50 mg & $3-5 \mathrm{mg} / \mathrm{kg} * *$ & $712.000 \mathrm{a} 1.246 .000$ \\
Caspofungina 50 mg & $50 \mathrm{mg}$ & 181.059
\end{tabular}

*Fuente: Revista Kairos, $\mathrm{N}^{\circ} 162$, julio 2003. Valor neto en pesos sin impuestos agregados. Sólo se consideraron fármacos originales. $* *$ Los datos de voriconazol fueron estimados de acuerdo a consultas del autor. 
comparados no supera los 300 en cada grupo. En la opinión de algunos expertos, esta combinación debe ser mirada aún como una aproximación experimental, de eficacia incierta y con riesgos potenciales $^{3,25,29}$.

\section{Combinaciones de antifúngicos y terapias asociadas}

El advenimiento de nuevos productos antifúngicos con diferentes mecanismos de acción ha puesto en escena el uso combinado de ellos. En particular se ha puesto énfasis en la combinación de anfotericina B y caspofungina por sus mecanismos complementarios, uno sobre la membrana y otro sobre la pared celular. Esta combinación ha demostrado un efecto sinérgico in vitro contra los géneros Aspergillus, Cryptococcus y Fusarium.

La experiencia acumulada sólo esta basada por ahora en series de casos con resultados favorables y promisorios. Esta combinación resultó efectiva para el rescate de pacientes con leucemia afectados por aspergilosis pulmonar sin una respuesta previa a anfotericina $\mathrm{B}$. Incluso se pudo observar una respuesta clínica en pacientes sin recuperación de la neutropenia ${ }^{30}$. Caspofungina también ha sido utilizada con éxito junto a itraconazol oral para tratar la misma condición en pacientes inmunosuprimidos ${ }^{31}$.

Las infecciones por $T$. beigelii parecen responder mejor ante una combinación de triazoles y anfotericina $\mathrm{B}$, a pesar de que los triazoles han sido utilizados con éxito en forma aislada ${ }^{32}$.

La eficacia clínica de anfotericina B convencional aumenta notoriamente cuando se acompaña de factores estimuladores de colonias (G-CSF) en el tratamiento de pacientes neutropénicos febriles con sospecha de infección fúngica y además permite un ahorro de recursos en comparación a un tratamiento sólo con anfotericina $\mathrm{B}$ convencional ${ }^{33}$.

\section{Aspectos farmacoeconómicos}

La disponibilidad de nuevos fármacos para el tratamiento de las infecciones fúngicas sistémicas ha incrementado el arsenal con el que se contaba hasta hace algunos pocos años y que estaba limitado a anfotericina $\mathrm{B}$, itraconazol en cápsulas y fluconazol en diferentes presentaciones. En ese escenario, los costos y los efectos adversos debían asumirse en forma obligada. La diversidad actual generada por diferentes alternativas de menor toxicidad ha hecho necesario evaluar los aspectos farmacoeconómicos de estas intervenciones.

Los costos directos asociados a un tratamien- to están compuestos por los valores de adquisición del producto, tratamientos de segunda línea ante fracaso o intolerancia, pruebas de laboratorio e intervenciones para el manejo de las complicaciones asociadas al tratamiento y los días de hospitalización. El costo del producto sólo representa una fracción del valor total.

Hasta la fecha sólo se dispone de estudios de farmacoeconomía para algunas formulaciones de anfotericina B asociadas a lípidos (excluyendo la mezcla con Intralipid®), fluconazol e itraconazol $\mathrm{ev}^{34,35}$. A pesar de que el riesgo de nefrotoxicidad disminuye con la utilización de anfotericina B liposomal, este tratamiento resulta más oneroso en términos globales que uno con el preparado convencional para el tratamiento de pacientes neutropénicos febriles con sospecha de infección ${ }^{35,36}$. Para una relación costo-efectiva equivalente a anfotericina B convencional, el costo de la forma liposomal debería ser inferior al $50 \%$ del precio actual ${ }^{35}$.

En las Tablas 6 y 7 se presentan los costos de adquisición de estos productos en E.U.A. y en Chile, respectivamente. Itraconazol ev y caspofungina poseen valores intermedios en comparación a los elevados precios de las formulaciones asociadas a lípidos de anfotericina B. A pesar de que no se dispone de información farmacoeconómica sobre caspofungina, el uso empírico de itraconazol ev ha resultado ser más costo-efectivo en el tratamiento de pacientes con neutropenia (con o sin trasplante de precursores hematopoyéticos) y sospecha de infección fúngica, en comparación a anfotericina $\mathrm{B}$ convencional $\mathrm{O}$ liposomal ${ }^{34}$.

En el tratamiento de pacientes no neutropénicos con candidemia, el tratamiento con anfotericina B convencional es más costo-efectivo que fluconazol ev, debido al mayor costo y la necesidad habitual de prolongar el tratamiento con este último ${ }^{34,37}$. El paso a fluconazol oral antes del séptimo día de tratamiento permite una relación costo-efectividad equivalente entre ambas alternativas ${ }^{34}$.

El uso de factores estimuladores de colonias (G-CSF a dosis de 3 a $5 \mu \mathrm{g} / \mathrm{kg} /$ día) ha demostrado ser una estrategia costo-efectiva en el tratamiento de pacientes neutropénicos febriles con sospecha de infección fúngica cuando es utilizada en conjunto con anfotericina B convencional $^{33}$. El alto precio de los factores estimuladores de colonias es compensado por los ahorros asociados a una mayor eficacia clínica, menor uso de esquemas de segunda línea y disminución en la estadía hospitalaria

Aunque no se disponga de estudios farmaco- 
Tabla 8. Factores a considerar en la selección de compuestos antifúngicos ante infecciones invasoras o diseminadas

\begin{tabular}{|c|c|}
\hline Variable & Comentarios \\
\hline - Tipo de microorganismo en tratamiento & $\begin{array}{l}\text { La sospecha de una infección por hongos filamentosos impide } \\
\text { la utilización de fluconazol, pero no contraindica la utilización } \\
\text { de otros triazólicos como voriconazol o itraconazol en condi- } \\
\text { ciones específicas. Anfotericina B y caspofungina también po- } \\
\text { seen actividad contra hongos filamentosos }\end{array}$ \\
\hline - Presencia de neutropenia & $\begin{array}{l}\text { Esta condición aumenta la probabilidad de infecciones por } \\
\text { hongos filamentosos, cuya demostración además es difícil. En } \\
\text { consecuencia debe asegurarse su cobertura en el tratamiento }\end{array}$ \\
\hline - Sitio de infección & $\begin{array}{l}\text { Los compuestos triazólicos son los de mejor penetración al } \\
\text { SNC (excepto itraconazol). La penetración de caspofungina es } \\
\text { mínima y algo mejor en el caso de anfotericina B }\end{array}$ \\
\hline - Asociación de la infección con un cuerpo extraño & $\begin{array}{l}\text { Las especies de Candida sintetizan biopolímeros que facilitan } \\
\text { la evasión de la respuesta inmune e impiden el efecto de los } \\
\text { antifúngicos. Deben ser removidos si están asociados a infec- } \\
\text { ción, en forma independiente a la selección del compuesto }\end{array}$ \\
\hline - Presencia de falla renal & $\begin{array}{l}\text { La existencia de insuficiencia renal obliga a ajustar las dosis de } \\
\text { fluconazol, contraindica el uso parenteral de itraconazol o } \\
\text { voriconazol y limita las posibilidades de usar anfotericina B }\end{array}$ \\
\hline - Presencia de falla hepática & $\begin{array}{l}\text { En casos severos contraindica el uso de triazólicos. No existe } \\
\text { experiencia con caspofungina en falla hepática severa }\end{array}$ \\
\hline - Potenciales interacciones con otros fármacos & $\begin{array}{l}\text { Numerosas interacciones en el caso de los compuestos } \\
\text { triazólicos. Mínimas en el caso de caspofungina. Algunos com- } \\
\text { puestos pueden aumentar la nefrotoxicidad de anfotericina B, } \\
\text { tales como ciclosporina y aminoglucósidos }\end{array}$ \\
\hline - Vía de administración necesaria & $\begin{array}{l}\text { Los compuestos triazólicos poseen una amplia versatilidad ya } \\
\text { que pueden ser administrados por vía oral o periférica. } \\
\text { Anfotericina B está asociada a flebitis y muchas veces requie- } \\
\text { re una administración por vía central. Caspofungina puede ser } \\
\text { administrada por vía endovenosa periférica }\end{array}$ \\
\hline - Requerimiento de otras modalidades terapéuticas & $\begin{array}{l}\text { La remoción del foco es un requisito fundamental en pacientes } \\
\text { con mucormicosis, abscesos cerebrales, endocarditis infeccio- } \\
\text { sa asociada a válvula protésica, infección asociada a un acceso } \\
\text { vascular y casos graves de endoftalmitis }\end{array}$ \\
\hline
\end{tabular}

económicos locales, es necesario recordar que el costo global de un tratamiento antifúngico se incrementa con la utilización de fármacos endovenosos versus compuestos orales, la necesidad de cubrir hongos filamentosos, la presencia o desarrollo de falla renal, la necesidad de utilizar esquemas de segunda línea y el uso empírico de ellos sin infección confirmada.

\section{Decisiones terapéuticas}

Los diferentes compuestos antifúngicos deben ser aplicados en escenarios clínicos variables, con ventajas y desventajas relativas. El conocimiento y la selección de la mejor opción son fundamentales en el uso de estos tratamientos.

En la decisión terapéutica participan varios factores que son importantes de analizar (Tabla 8). Por ejemplo, en pacientes médico-quirúrgicos no neutropénicos rara vez se observan infecciones por hongos filamentosos y los fármacos de elección corresponden a los triazoles. En contraste, en pacientes neutropénicos la probabilidad de infecciones por este tipo de hongos aumenta y debe siempre contemplarse una alternativa activa contra ellos. La existencia de insuficiencia renal o hepática restringe el uso de compuestos específicos y la penetración al SNC no es similar para todas las alternativas. Para cierto tipo de infecciones, la remoción quirúrgica del foco es fundamental. La selección de una alternativa específica tiene también importantes implicancias económicas.

En la Tabla 9 se presentan las alternativas 
Tabla 9. Alternativas de tratamiento en infecciones fúngicas diseminadas o invasoras

\begin{tabular}{|c|c|c|}
\hline Tipo de infección & Alternativas de tratamiento* & Comentarios \\
\hline $\begin{array}{l}\text { Candidiasis diseminada o } \\
\text { infecciones invasoras asociadas } \\
\text { a C. albicans (en ausencia de } \\
\text { neutropenia) }\end{array}$ & Fluconazol & $\begin{array}{l}\text { Evidencia proveniente de ensayos } \\
\text { randomizados }\end{array}$ \\
\hline $\begin{array}{l}\text { Infecciones del SNC asociadas } \\
\text { a C. albicans }\end{array}$ & $\begin{array}{l}\text { Fluconazol en altas dosis, } \\
\text { anfotericina B o voriconazol }\end{array}$ & $\begin{array}{l}\text { Caspofungina no logra una pene- } \\
\text { tración adecuada al SNC }\end{array}$ \\
\hline Endoftalmitis por C. albicans & Fluconazol & $\begin{array}{l}\text { Los casos más graves requieren } \\
\text { vitrectomía o dosis intravítreas }\end{array}$ \\
\hline $\begin{array}{l}\text { Infecciones diseminadas o } \\
\text { invasoras asociadas a } \\
\text { Candida no albicans }\end{array}$ & Fluconazol, anfotericina B o voriconazol & $\begin{array}{l}\text { C. glabrata presenta CIM superio- } \\
\text { res a las observadas en } C \text {. albicans } \\
\text { que pueden ser resueltas con un } \\
\text { aumento de la dosis. } C \text {. krusei es } \\
\text { susceptible a anfotericina B, caspo- } \\
\text { fungina, voriconazol e itraconazol }\end{array}$ \\
\hline Aspergilosis pulmonar invasora & Anfotericina B o voriconazol & $\begin{array}{l}\text { Voriconazol y caspofungina repre- } \\
\text { sentan alternativas validadas pero } \\
\text { de mayor costo }\end{array}$ \\
\hline $\begin{array}{l}\text { Sospecha de infección fúngica } \\
\text { invasora en pacientes con } \\
\text { neutropenia febril }\end{array}$ & $\begin{array}{l}\text { Anfotericina } \mathrm{B} \text {, itraconazol ev } \\
\text { o caspofungina }^{38}\end{array}$ & $\begin{array}{l}\text { Voriconazol podría ser } \\
\text { inferior a anfotericina } \mathrm{B}\end{array}$ \\
\hline Meningitis criptococóccica & $\begin{array}{l}\text { Anfotericina B en pacientes de } \\
\text { alto riesgo y fluconazol en pacientes } \\
\text { de bajo riesgo en la fase de inducción }\end{array}$ & $\begin{array}{l}\text { Fluconazol representa el fármaco } \\
\text { de elección en el tratamiento de } \\
\text { consolidación y en profilaxis se- } \\
\text { cundaria }\end{array}$ \\
\hline Mucormicosis & Anfotericina B & $\begin{array}{l}\text { Otros antifúngicos no poseen ac- } \\
\text { tividad contra este tipo de agente. } \\
\text { La resección quirúrgica es necesa- } \\
\text { ria en todos los casos }\end{array}$ \\
\hline \multicolumn{3}{|l|}{ Requieren ajuste de dosis } \\
\hline $\begin{array}{l}\text { Infecciones por levaduras } \\
\text { en presencia de falla renal }\end{array}$ & Fluconazol a dosis ajustadas & $\begin{array}{l}\text { Considerar otro compuesto como } \\
\text { caspofungina ante aislados de } C \text {. } \\
\text { glabrata o } C \text {. krusei }\end{array}$ \\
\hline $\begin{array}{l}\text { Infecciones por hongos } \\
\text { filamentosos en presencia } \\
\text { de falla renal }\end{array}$ & Caspofungina & $\begin{array}{l}\text { Voriconazol endovenoso está con- } \\
\text { traindicado con filtración glomeru- } \\
\text { lar }<50 \mathrm{ml} / \mathrm{min}\end{array}$ \\
\hline $\begin{array}{l}\text { Infecciones por levaduras en } \\
\text { presencia de falla hepática }\end{array}$ & Anfotericina B & $\begin{array}{l}\text { Triazólicos no se consideran apro- } \\
\text { piados por su metabolismo hepá- } \\
\text { tico e interacción con otros fárma- } \\
\text { cos. No existe experiencia con cas- } \\
\text { pofungina en pacientes con falla } \\
\text { hepática severa }\end{array}$ \\
\hline $\begin{array}{l}\text { Infecciones por hongos } \\
\text { filamentosos en presencia } \\
\text { de falla hepática }\end{array}$ & $\begin{array}{l}\text { Anfotericina } \mathrm{B} \text {, caspofungina en } \\
\text { pacientes con falla hepática leve o } \\
\text { moderada }\end{array}$ & Idem \\
\hline
\end{tabular}

* En opinión del autor 
terapéuticas para situaciones clínicas específicas que resumen los contenidos de esta revisión.

\section{Bibliografía}

1.- Fica A. Tratamiento de infecciones fúngicas sistémicas. Parte I: Fluconazol, itraconazol y voriconazol. Rev Chil Infect 2004; 21: 28-38.

2.- Diomedi A. Nuevos antifúngicos: las equinocandinas. Rev Chil Infect 2004; 21: 89-101.

3.- Thipine M, Letscher-Bru V, Herbrecht R. Amphotericin $\mathrm{B}$ and its new formulations: pharmacologic characteristics, clinical efficacy, and tolerability. Transpl Infect Dis 1999; 1: 273-83.

4.- Stone E A, Fung H B, Kirschenbaum H L. Caspofungin: An echinocandin antifungal agent. Clin Ther 2002; 24: 351-77.

5.- Espinel-Ingroff A. Comparison of in vitro activities of the new triazole SCH 56592, and the echinocandins MK-0991 (L 743,872) and LY303366 against opportunistic filamentous and dimorphic fungi and yeasts. J Clin Microbiol 1998; 36: 2950-6.

6.- Ernst E J, Klepser M E, Pfaller M A. Postantifungal effects of echinocandin, azole, and polyene antifungal agents against Candida albicans and Cryptococcus neoformans. Antimicrob Agents Chemother 2000; 44: 1108-11.

7.- Gotzsche P C, Johansen H K. Meta-analysis of prophylactic or empirical antifungal treatment versus placebo or no treatment in patients with cancer complicated by neutropenia. Br Med J 1997; 314: 1238-44.

8.- Ellis M. Amphotericin B preparations: a maximum tolerated dose in severe invasive fungal infections? Transpl Infect Dis 2000; 2: 51-61.

9.- Dupont B. Overview of the lipid formulations of amphotericin B. J Antimicrob Chemother 2002; 49, Suppl. S1: 31-6.

10.- Branch R A. Prevention of amphotericin B-induced renal impairment. Arch Intern Med 1988; 148: 2389-94.

11.- Eriksson U, Seifert B, Schaffner A. Comparison of effects of amphotericin B deoxycholate infused over 4 hours or 24 hours: randomized controlled trial. $\mathrm{Br}$ Med J 2001; 322: 1-6.

12.- Oldfield III E C, Garst P D, Hostettler C, White M, Samuelson D. Randomized, double-blind trial of 1- versus 4-hour amphotericin B infusion durations. Antimicrob Agents Chemother 1990; 34: 1402-6.

13.- Llanos A, Cieza J, Bernardo J, Echevarria J, Biaggioni I, Sabra R, et al. Effect of salt supplementation on amphotericin B nephrotoxicity. Kidney Int 1991; 40 (2): 302-8.

14.- Bowden R, Chandrasekar P, White M H, Li X, Pietrelli L, Gurwith M, et al. A double-blind, randomized, controlled trial of amphotericin B colloidal dispersion versus amphotericin B for treatment of invasive aspergillosis in immunocompromised patients. Clin Infect Dis 2002; 35 (4): 359-66.

15.- Dismukes W E. Introduction to antifungal drugs. Clin Infect Dis 2000; 30: 653-7.

16.- Shadkan Y, Segal E, Bor A, Gov Y, Rubin M, Lichtenberg D. The use of commercially available lipid emulsions for the preparation of amphotericin B-lipid admixtures. J Antimicrob Chemother 1997; 39: 6558.

17.- Walker S, Tailor SAN, Lee M, Louie L, Luoie M, Simor AE. Amphotericin B in lipid emulsion: stability, compatibility, and in vitro antifungal activity. Antimicrob Agents Chemother 1998; 42: 762-6.

18.- Ayestaran A, López R M, Montoro J B, Estibalez A, Pou L, Julia A, et al. Pharmacokinetics of conventional formulation versus fat emulsion formulation of amphotericin B in a group of patients with neutropenia. Antimicrob Agents Chemother 1996; 40 (3): 609-12.

19.- Chavanet P Y, Garry I, Charlier N, Caillot D, Kisterman J P, D'Athis M, et al. Trial of glucose versus fat emulsion in preparation of amphotericin for use in HIV infected patients with candidiasis. $\mathrm{Br}$ Med J 1992; 305 (6859): 921-5.

20.- Moreau P, Milpied N, Fayette N, Ramee J F, Harousseau J L. Reduced renal toxicity and improved clinical tolerance of amphotericin B mixed with intralipid compared with conventional amphotericin $\mathrm{B}$ in neutropenic patients. J Antimicrob Chemother 1992; 30: 535-41.

21.- Caillot D, Reny G, Solary E, Casasnovas O, Chavanet P, Bonnotte B, et al. A controlled trial of the tolerance of amphotericin B infused in dextrose or in intralipid in patients with haematological malignancies. J Antimicrob Chemother 1994; 33 (3): 603-13.

22.- Pascual B, Ayestaran A, Montoro J B, Oliveras J, Estibalez A, Julia A, et al. Administration of lipidemulsion versus conventional amphotericin $\mathrm{B}$ in patients with neutropenia. Ann Pharmacother 1995; 29 (12): 1197-201.

23.- Joly V, Aubry P, Ndayiragide A, Carriere I, Kawa E, Mlika-Cabanne N, et al. Randomized comparison of amphotericin B deoxycholate dissolved in dextrose or intralipid for the treatment of AIDS-associated cryptococcal meningitis. Clin Infect Dis 1996; 23 (3): 556-62.

24.- Sorkine P, Nagar H, Weinbroum A, Setton A, Israitel E, Scarlatt A, et al. Administration of amphotericin B in lipid emulsion decreases nephrotoxicity: results of a prospective, randomized, controlled study in critically ill patients. Crit Care Med 1996; 24 (8): 1311-5.

25.- Schoffski P, Freund M, Wunder R, Petersen D, Kohne C H, Hecker H, et al. Safety and toxicity of amphotericin $\mathrm{B}$ in glucose $5 \%$ or intralipid $20 \%$ in neutropenic patients with pneumonia or fever of unknown origin: randomised study. Br Med J 1998; 317 (7155): 37984.

26.- Barquist E, Fein E, Shadick D, Johnson J, Clark J, Shatz D. A randomized prospective trial of amphotericin B lipid emulsion versus dextrose colloidal solution in critically ill patients. J Trauma 1999; 47: 336-40.

27.- Nucci M, Loureiro M, Silveira F, Casali A R, Bouzas L F, Velasco E, et al. Comparison of the toxicity of amphotericin B in 5\% dextrose with that of amphotericin $\mathrm{B}$ in fat emulsion in a randomized trial with cancer patients. Antimicrob Agents Chemother 1999; 43 (6): 1445-8.

28.- Nath C E, Shaw P J, Gunning R, McLachlan A J, Earl $\mathrm{J}$ W. Amphotericin B in children with malignant diseases: a comparison of the toxicities and pharmacokinetics of amphotericin B administered in dextrose versus lipid emulsion. Antimicrob Agents Chemother 1999; 43: 1417-23.

29.- Sievers T M, Kubak B, Wong-Beringer A. Safety and efficacy of intralipid emulsions of amphotericin B. J Antimicrob Chemother 1996; 38: 333-47.

30.- Aliff T B, Maslak P G, Jurcic J G, Heaney M L, Cathcart K N, Sepkowitz K A, et al. Refractory Asper- 
gillus pneumonia in patients with acute leukemia: Successful therapy with combination caspofungin and liposomal amphotericin. Cancer 2003; 97 (4): 1025-32.

31.- Rubin M A, Carroll K C, Cahill B C. Caspofungin in combination with itraconazole for the treatment of invasive aspergillosis in humans. Clin Infect Dis 2002; 34: 1160 .

32.- Goodman D, Pamer E, Jakubowski A, Morris C, Sepkowitz K. Breakthrough trichosporinosis in a bone marrow transplant recipient receiving caspofungin acetate. Clin Infect Dis 2002; 35: 35-6.

33.- Flynn T N, Kelsey S M, Hazel D L, Guest J F. Cost effectiveness of amphotericin B plus G-CSF compared with amphotericin B monotherapy. Treatment of presumed deep-seated fungal infection in neutropenic patients in UK. Pharmacoeconomics 1999; 16 (5 Pt 2): 543-50.

34.- Van Gool R. The cost of treating systemic fungal infections. Drugs 2001; 61 Suppl 1: 49-56.

35.- Cagnoni $\mathrm{P} J$, Walsh $\mathrm{T} \mathrm{J}$, Prendergast $\mathrm{M} \mathrm{M}$,
Bodensteiner D, Hiemenz S, Greenberg R N, et al. Pharmacoeconomic analysis of liposomal amphotericin B versus conventional amphotericin B in the empirical treatment of persistently febrile neutropenic patients. J Clin Oncol 2000; 18 (12): 2476-83.

36.- Boogaerts M, Tormans G, Maes E, et al. Costeffectiveness analysis of Ambisome (AMB) vs amphotericin B (AMPHOB) in the empiric treatment of febrile neutropenia in adults and children. Blood 1996; 88 Suppl 1: 501A.

37.- Dranitsaris G, Phillips P, Rotstein C, Puodziunas A, Shafran S, Garber G, et al. Economic analysis of fluconazole versus amphotericin B for the treatment of candidemia in non-neutropenic patients. Pharmacoeconomics 1998; 13 (5): 509-18.

38.- Walsh TJ, Teppler H, Donowitz GR, Maertens JA, Boden RL, Dmoszynska A, et al. Caspofungin versus liposomal amphotericin B for empirical antifungal therapy in patients with persistent fever and neutropenia. N Engl J Med 2004; 351: 1391-402.

Correspondencia a:

Alberto Fica Cubillos

afica@ns.hospital.uchile.cl 